\title{
Prostate cancer recurrence: can PSA guide imaging?
}

\author{
P. Mapelli ${ }^{1}$ V. Panebianco ${ }^{2}$ Maria Picchio ${ }^{1}$
}

Received: 15 May 2015 / Accepted: 19 May 2015 / Published online: 9 July 2015

(C) Springer-Verlag Berlin Heidelberg 2015

\section{Key points}

- PSA measurement does not discriminate the site and extent of recurrent disease

- Imaging may be of help in detecting site and extent of recurrence

- Choline PET/CT is recommended in patients with PCa recurrence and $P S A \geq 1 \mathrm{ng} / \mathrm{mL}$

- Mp-MRI is recommended for PSA between 0.2 and $1 \mathrm{ng} /$ $m L$ to detect small local recurrence

- The appropriate use of imaging modalities can guide personalized treatment

- PSMA might overcome some of the limitations of choline in PCa recurrence assessment

The relevance of prostate cancer (PCa) as a clinical problem is clearly and simply represented by numbers: according to the International Agency for Research on Cancer, the worldwide PCa burden is expected to grow to 1.7 million new cases and 499,000 deaths annually by 2030 due to the growth and ageing of the global population [1]. Moreover, prostate-specific antigen (PSA) measurement for early detection and monitoring of men with $\mathrm{PCa}$ has increased the number of patients presenting with potentially curable disease.

Maria Picchio

picchio.maria@hsr.it

1 Department of Nuclear Medicine, IRCCS San Raffaele Scientific Institute, Milan, Italy

2 Department of Radiological Sciences, Oncology and Pathology, Sapienza University of Rome, Rome, Italy
Although many treatment options are available for localized disease, between $27 \%$ and $53 \%$ of patients treated for primary $\mathrm{PCa}$ will experience a recurrence, typically indicated by a rise in PSA serum levels. Once recurrence is suspected, the discrimination between local and distant relapse is essential in choosing the best treatment strategy. If restricted to PSA measurement, which is limited by the lack of serum biomarkers, and is unable to discriminate the precise recurrence site and extent, the assessment of disease progression is definitely suboptimal. However, it has been shown that PSA kinetics including PSA doubling time and PSA velocity strongly correlate with clinical outcomes after local therapy and may suggest the likelihood of a patient having occult distant metastatic disease [2].

Considering that $\mathrm{PCa}$ recurrence can occur at the locoregional level or involve lymph nodes and bone, ideally a one-stop-shop imaging modality able to identify all the possible sites of recurrence in a single examination would be an invaluable diagnostic weapon. But what are the best means currently available for clinicians to properly identify and characterize PCa recurrence? Is PSA able to indicate the best diagnostic modality to be performed?

Transrectal ultrasonography (TRUS) is not sufficiently sensitive or specific for the early detection of PCa local recurrence, it is invasive and expensive, and additionally it has a low but not insignificant rate of complications such as infections. Especially in patients with low PSA levels, TRUS is not the optimal choice and, moreover, multiple biopsies are often required because of potential sampling error that cannot definitely exclude local recurrence. Because of these limitations, TRUS and biopsy are only recommended if they will affect the treatment plan, not being necessary before second-line therapy in most patients [3].

Over recent years, the development of imaging techniques combining anatomical, functional and biological information 
that are able to detect metabolic changes in cellular metabolism has significantly improved the early detection of $\mathrm{PCa}$ recurrence. In particular, PET/CT and multiparametric MRI (mp-MRI) have provided substantial and crucial advances in the detection and localization of both local and distant $\mathrm{PCa}$ recurrence [4].

The use of PET/CT with choline labelled either with ${ }^{11} \mathrm{C}$ or ${ }^{18} \mathrm{~F}$ is still controversial for $\mathrm{PCa}$ diagnosis, due to the possible false-positive distribution of choline in some benign conditions such as hyperplasia and prostatitis; however, in patients with PCa recurrence, this imaging modality is largely used by clinicians to assess disease extension. Being a whole-body examination, choline PET/CT is able to accurately acquire metabolic and anatomical imaging in a single step, identifying the site of recurrent disease in patients with biochemical failure after radical primary treatment. Its role in restaging $\mathrm{PCa}$ has been investigated in some depth, and the most important information arising from this work includes the fact that the detection rate, and so the sensitivity, of this technique is tightly connected with PSA serum level: the higher the PSA level at recurrence, the higher is the positive detection rate of $\mathrm{PET} / \mathrm{CT}$. Moreover, correlations between the detection rate of choline PET/CT and PSA kinetics have also been reported $[5,6]$.

Does this mean that there is a specific PSA cut-off value that can be used to discriminate patients who will benefit from PET/CT and others who could potentially be incorrectly restaged? Several studies have investigated the possible usefulness of using a specific cut-off value to define the effective clinical benefit of choline PET/CT in PCa restaging. The vast majority of those who have studied this topic agree that the clinical indication for using choline PET/CT in patients with PCa recurrence is a PSA serum level higher than $1 \mathrm{ng} / \mathrm{mL}$ at presentation. In such patients choline PET/CT shows its best sensitivity and specificity in detecting lymph node and distant metastases. Its accuracy in detecting locoregional PCa recurrence is mainly influenced by lesion size and is usually higher for lesions larger than $1 \mathrm{~cm}$. Following these considerations on choline PET/CT, the question arises: what about those patients experiencing a PCa biochemical relapse with a PSA value lower than $1 \mathrm{ng} / \mathrm{mL}$ and in whom the exclusion of a locoregional recurrence is essential in planning the most appropriate treatment?

Currently, mp-MRI and specifically mp-MRI dynamic contrast-enhanced MRI (DCE MRI) in addition to morphological (T2-W MR), diffusion imaging (DW MRI) and sometimes ${ }^{1} \mathrm{H}$ MR spectroscopy (MRSI) are the most reliable techniques for detection of PCa locoregional recurrence. In patients with only a mild elevation in PSA (ranging between 0.2 and $1 \mathrm{ng} / \mathrm{ml}$ ) at recurrence, who most frequently experience only a local recurrence, mp-MRI is clearly the imaging modality of choice. MRI, thanks to its inherently superior contrast and spatial resolution, especially with an endorectal coil, is an emerging and promising modality for the evaluation of the prostatic fossa after radical prostatectomy [7]. In addition, the recent development of functional MRI techniques has provided promising results for accurate detection and characterization of small recurrences of $\mathrm{PCa}(<1 \mathrm{~cm})$. Moreover, mpMRI after radical prostatectomy is a very useful tool to discriminate between locoregional relapse and small amounts of residual healthy glandular tissue, scar/fibrosis and granulation tissue, and it may even be able to assess the aggressiveness of nodule recurrence by means of apparent diffusion coefficient values using the diffusion technique. In these patients mpMRI can avoid false-positive results that could occur with choline PET/CT.

Currently mp-MRI is recommended when PSA values are between 0.2 and $1 \mathrm{ng} / \mathrm{mL}$ to detect even small local recurrence [8]. What is the clinical impact of mp-MRI? In patients scheduled for local salvage external beam radiotherapy after radical prostatectomy, accurate anatomical localization of tumour deposits in the prostate bed after prostatectomy may allow individualization of the field of irradiation, thereby maximizing efficacy and minimizing toxicity to normal surrounding tissues. In this setting mp-MRI findings could be used to apply a stereotactic boost to the recurrence site, potentially improving the control of local disease and avoiding further locoregional relapses over time. Furthermore, the differential diagnosis between residual healthy glandular tissue and locoregional neoplastic recurrence is of paramount importance for the radiation oncologist because the dose of radiation delivered to the prostate bed is quite different. However, the possibility of use mpMRI to efficiently restage $\mathrm{PCa}$ recurrence is hampered by the fact that this modality is not a whole-body technique and so the presence of distant metastases can be underestimated if imaging is limited to the sole use of mp-MRI.

So, can PSA guide morphofunctional imaging in PCa recurrence? According to available data, PET/CT is recommended for patients with a PSA value higher than $1 \mathrm{ng} / \mathrm{mL}$ because of its accuracy in detecting lymph node and distant metastases in a single session examination, while mp-MRI is indicated in patients with a biochemical failure lower than $1 \mathrm{ng} / \mathrm{mL}$ because of its higher accuracy in detecting locoregional recurrence. This splitting of the most appropriate clinical use of choline PET/CT and mp-MRI in the detection of PCa recurrence on the basis of PSA values might be overcome by the new PET/MRI hybrid scanners that have recently been introduced in clinical practice [9]. This new technique that combines simultaneous acquisition of mp-MRI and PET images and provides combined structural, metabolic and functional imaging information in a whole-body single session examination is currently paving the way to a new more efficient and accurate approach to the detection of $\mathrm{PCa}$ recurrence. Indeed, the possibility of combining two examinations in a single session and the whole-body nature of this hybrid modality are of crucial relevance in the clinical setting because this modality could be used to choose the most appropriate 
treatment strategy in a less time-consuming and reasonably accurate way.

Finally, although choline is the most commonly used and specific PET radiotracer for investigating PCa recurrence, alternative radiocompounds have been tested with the aim of overcoming some of the limitations of choline in this setting. Prostatespecific membrane antigen (PSMA), that targets the internal domain of the PSMA receptor, is one of the most promising, showing a better signal to background ratio than choline, with higher sensitivity in lesion detection, including small lymph node, central bone and liver metastases, and also in patients with low PSA levels $[10,11]$. In particular, PSMA is labelled with ${ }^{68} \mathrm{Ga}$, and thus its clinical application does not require an on-site cyclotron because it can be extracted from a commercially available ${ }^{68} \mathrm{Ge} /{ }^{68} \mathrm{Ga}$ radionuclide generator, in contrast to radiolabelled choline tracers which require isotopes produced by a cyclotron.

In conclusion, although PSA still remains the first clinical weapon used to identify PCa recurrence, we suggest that its fundamental application is to indicate the best imaging modality for a proper and accurate restaging of PCa patients and so to guide the choice of the most appropriate personalized treatment.

\section{References}

1. Ferlay J, Shin HR, Bray F, Forman D, Mathers C, Parkin DM. Estimates of worldwide burden of cancer in 2008: GLOBOCAN 2008. Int J Cancer. 2010;127:2893-917.

2. Lee AK, D'Amico AV. Utility of prostate-specific antigen kinetics in addition to clinical factors in the selection of patients for salvage local therapy. J Clin Oncol. 2005;23:8192-7.
3. Heidenreich A, Bastian PJ, Bellmunt J, Bolla M, Joniau S, van der Kwast T, et al. EAU guidelines on prostate cancer. Part II: treatment of advanced, relapsing, and castration-resistant prostate cancer. Eur Urol. 2014;65:467-79.

4. Picchio M, Mapelli P, Panebianco V,Castellucci P, Incerti E, Briganti A, et al. Imaging biomarkers in prostate cancer: role of PET/CT and MRI. Eur J Nucl Med Mol Imaging. 2015;42(4):644-55.

5. Picchio M, Briganti A, Fanti S, Heidenreich A, Krause BJ, Messa $\mathrm{C}$, et al. The role of choline positron emission tomography/ computed tomography in the management of patients with prostate-specific antigen progression after radical treatment of prostate cancer. Eur Urol. 2011;59:51-60.

6. Giovacchini G, Picchio M, Garcia-Parra R, Mapelli P, Briganti A, Montorsi $\mathrm{F}$, et al. [11C]choline positron emission tomography/ computerized tomography for early detection of prostate cancer recurrence in patients with low increasing prostate specific antigen. J Urol. 2013;189:105-10.

7. Panebianco V, Barchetti F, Sciarra A, Musio D, Forte V, Gentile V, et al. Prostate cancer recurrence after radical prostatectomy: the role of 3-T diffusion imaging in multi-parametric magnetic resonance imaging. Eur Radiol. 2013;23:1745-52.

8. Panebianco V, Barchetti F, Musio D, De Felice F, Proietti C, Indino EL, et al. Advanced imaging for the early diagnosis of local recurrence prostate cancer after radical prostatectomy. Biomed Res Int. 2014;2014:827265.

9. de Perrot T, Rager O, Scheffler M, Lord M, Pusztaszeri M, Iselin C, et al. Potential of hybrid 18F-fluorocholine PET/ MRI for prostate cancer imaging. Eur J Nucl Med Mol Imaging. 2014;41:1744-55.

10. Afshar-Oromieh A, Zechmann CM, Malcher A, Eder M, Eisenhut $\mathrm{M}$, Linhart HG, et al. Comparison of PET imaging with a (68)Galabelled PSMA ligand and (18)F-choline-based PET/CT for the diagnosis of recurrent prostate cancer. Eur J Nucl Med Mol Imaging. 2014;41:11-20.

11. Yu CY, Desai B, Ji L, Groshen S, Jadvar H. Comparative performance of PET tracers in biochemical recurrence of prostate cancer: a critical analysis of literature. Am J Nucl Med Mol Imaging. 2014;4:580-601. 